\title{
Thermal annealing and evolution of defects in neutron-irradiated cubic $\mathrm{SiC}$
}

\author{
V.Ya. Bratus', R.S. Melnyk, B.D. Shanina, S.M. Okulov \\ V. Lashkaryov Institute of Semiconductor Physics, NAS of Ukraine, \\ 45, prospect Nauky, 03680 Kyiv, Ukraine \\ e-mail:v_bratus@isp.kiev.ua,melnyk_rs@yahoo.com,shanina_bela@rambler.ru,okulov@isp.kiev.ua
}

\begin{abstract}
A careful study of neutron-irradiated cubic SiC crystals (3C-SiC $\langle n\rangle)$ has been performed using electron paramagnetic resonance (EPR) in the course of their thermal annealing within the $200 \ldots 1100^{\circ} \mathrm{C}$ temperature range. Several inherent temperatures have been found for annealing and transformations of primary defects in $3 \mathrm{C}$-SiC $\langle n\rangle$ among which there are isolated negatively charged silicon vacancy $\mathrm{V}_{\mathrm{Si}}{ }^{-}$, neutral divacancy $\left(\mathrm{V}_{\mathrm{Si}}-\mathrm{V}_{\mathrm{C}}\right)^{0}$, negatively charged carbon vacancy-antisite pair $\left(\mathrm{V}_{\mathrm{C}}-\mathrm{C}_{\mathrm{Si}}\right)^{-}$and neutral carbon $\langle 100\rangle$ split interstitial $(\mathrm{CC})_{\mathrm{C}}{ }^{0}$. It has been shown that transformation of $\mathrm{V}_{\mathrm{Si}}{ }^{-}$into $\left(\mathrm{V}_{\mathrm{C}}-\mathrm{C}_{\mathrm{Si}}\right)^{-}$complex is among the mechanisms of silicon vacancy annealing. As it has been established on the basis of the observed hyperfine structure, the secondary T6 center is characterized by the fourfold silicon coordination and assigned to the spin $\mathrm{S}=3 / 2$ carbon vacancy-related pair defect. The symmetry reduction of the $\left(\mathrm{V}_{\mathrm{C}}-\mathrm{V}_{\mathrm{Si}}\right)^{0}$ center is attributed to local rearrangements in the neighborhood of divacancy, and its intensity variations are assigned to changes of the Fermi-level position. Two defects with similar symmetry and close values of zero-field splitting constants $D$, which concentrations increase by a factor of ten after annealing at $900{ }^{\circ} \mathrm{C}$, are tentatively attributed to the $\langle 100\rangle$ split interstitial $(\mathrm{CC})_{\mathrm{C}}{ }^{0}$ and $(\mathrm{NC})_{\mathrm{C}}{ }^{0}$ pairs.
\end{abstract}

Keywords: paramagnetic defect, EPR, neutron irradiation, silicon carbide, defect annealing, zero-field splitting.

Manuscript received 02.06.15; revised version received 09.09.15; accepted for publication 28.10.15; published online 03.12.15.

\section{Introduction}

The special features of silicon carbide polytypes, namely: wide bandgap, large thermal conductivity, high mobility of carries and breakdown electric field [1], enable to consider them as one of the most promising materials for high-power, high-frequency and hightemperature electronics [2]. Application of $\mathrm{SiC}$ devices for radiation-resistant and high-temperature operation necessitates detailed knowledge of intrinsic and irradiation damage defects and their thermal stability. Primary defects generated by high-energy particles in binary $\mathrm{SiC}$ compounds are vacancies, interstitials,
Frenkel pairs and antisites. Comprehensive information concerning the microscopic structure of defects can be extracted using investigations with magnetic resonance techniques like electron paramagnetic resonance (EPR), electron-nuclear double resonance (ENDOR) and optically detected magnetic resonance [3]. As a general rule, in these investigations a tentative model of a defect is based on the analysis of its spin-Hamiltonian parameters, mainly, the fine and hyperfine (HF) structure of EPR spectra. As of now, to verify the proposed model the first-principle calculations of the spin state, symmetry and hyperfine parameters for an assumed charge state are widely used [4]. 
Mention should be made of a renewed interest in the high-spin defects in SiC since they can be used as solidstate quantum bits even at room temperature along with the nitrogen-vacancy pair center in diamond [5]. Among the paramagnetic defects found in $\mathrm{SiC}$, a great deal of high-spin $S=1$ and $S=3 / 2$ centers have been detected after electron as well as neutron, proton and $\alpha$-particle irradiation [6-10]. For hexagonal polytypes of $\mathrm{SiC}$, the vacancy-related defects are reviewed in [11]. The microscopic structure of isolated negatively charged silicon vacancy $V_{\mathrm{Si}}^{-}$having $T_{d}$ symmetry has been identified with confidence in $3 \mathrm{C}, 4 \mathrm{H}$ and $6 \mathrm{H}$ polytypes of $\mathrm{SiC}[8,9$, 12], its high spin state $S=3 / 2$ has been determined with additional ENDOR study [8]. The negative- $U$ behavior has been found for spin $S=1 / 2$ negative carbon vacancy $\left(\mathrm{V}_{\mathrm{C}}{ }^{-}\right)$at both hexagonal and quasi-cubic lattice sites [13]. Almost ten spin-one defects with parameters closely related to that of irradiated crystals have been revealed after quenching and thermal treatment of $6 \mathrm{H}-\mathrm{SiC}$ crystals [14]. Nevertheless, the microscopic structure for many of them is still discussed.

Considerably less information about high-spin defects has been obtained in cubic SiC. Apart from the spin $S=3 / 2$ negatively charged silicon monovacancy, the so-called $T 1$ center, $T 6$ and $T 7$ centers were proposed to be some forms of spin-one vacancy-interstitial pairs after electron irradiation [12]. The LEl center with the spin $S=3 / 2$ detected after low-temperature electron irradiation was assigned to the Frenkel pair of silicon vacancy $V_{\mathrm{Si}}$ and the second neighbor silicon interstitial $\mathrm{Si}_{i}$ in the $3^{+}$charge state [15]. Noteworthy also are the numerous theoretical studies of damage recovery in cubic $\mathrm{SiC}$ that revealed various defect migration pathways with the energy barriers lower than $1 \mathrm{eV}$ (see, e.g., $[16,17]$ and references therein). But there is only little direct experimental information concerning these aspects as yet.

Neutron irradiation attracts attention of researchers with the potentialities for creating spin-one [7] and extended defects [18]. In our previous study [19], it has been shown that the $\mathrm{T} 1$ center and the spin-one neutral silicon-carbon divacancy $\left(V_{\mathrm{Si}}-V_{\mathrm{C}}\right)^{0}$, the $K y 5$ center, are the dominant primary defects in relatively high-dose neutron-irradiated 3C-SiC bulk crystals. In this work, the results of EPR studying the thermal annealing influence on radiation damage in these samples have been reported. Some preliminary results of this study have already been presented in [20].

\section{Experimental}

Unintentionally doped $n$-type cubic SiC single crystals were grown by thermal decomposition of methyltrichlorosilane in hydrogen [21], the concentration of the unavoidable nitrogen impurity was no more than $10^{17} \mathrm{~cm}^{-3}$. The crystals were irradiated at room temperature by reactor neutrons with the dose close to $10^{19} \mathrm{~cm}^{-2}$, in what follows they will be designated as
3C-SiC $\langle n\rangle$. Owing to high neutron penetration into $\mathrm{SiC}$ [22], the distribution of generated defects may be considered as uniform and homogeneous.

Post-irradiation isochronal thermal annealing procedures were carried out for 10 minutes within the 200 to $1100{ }^{\circ} \mathrm{C}$ temperature range in ambient gaseous He. The EPR study of defects was carried out within the temperature range $77 \ldots 300 \mathrm{~K}$ in the $\mathrm{X}$-band (microwave frequency $v_{m w} \sim 9.32 \ldots 9.45 \mathrm{GHz}$ ). The angular variations of EPR spectra were measured for a rotation of the magnetic field in the $(1 \overline{1} 0)$ crystal plane. The concentration of defects was determined with EPR by comparison with a $\mathrm{MgO}: \mathrm{Mn}^{2+}$ reference sample that contained the known number of spins. To estimate quantitatively the $g$-value and concentration of irradiation-induced defects, the studied sample and reference one were placed together into a microwave resonator. The number of defects was determined by comparing the double integrals of the first derivatives of the absorption signals registered on the investigated and reference specimens. The $g$-values of defects were determined with the accuracy $\Delta g= \pm 0.0001$ by using a microwave frequency counter and calibrating the magnetic field with a proton NMR probe.

\section{Results and discussion}

\section{A. Annealing of the negatively charged silicon monovacancy}

Fig. 1 shows the EPR spectra of a $3 \mathrm{C}-\mathrm{SiC}\langle n\rangle$ sample measured at $300 \mathrm{~K}$ before and after step-by-step thermal annealing with the magnetic field direction parallel to the $\langle 111\rangle$ crystal axis. The as-irradiated unannealed sample demonstrates an isotropic spectrum with the $g$ value of 2.0029 and four sets of superhyperfine (SHF) doublets with splitting typical to the $T 1$ center [12] superimposed by a broad more intense structureless slightly anisotropic background line BG. Components of the $T 1$ spectrum are broadened when comparing with those in electron-irradiated samples [12] due to a high concentration of this and additional defects making contribution to the $B G$ spectrum. The total concentration of paramagnetic defects determined by EPR is equal to $2.7 \cdot 10^{19} \mathrm{~cm}^{-3}$, exhibiting the high production rate of neutron irradiation for 3C-SiC, no less than $2.7 \mathrm{defects} / \mathrm{cm}^{3}$ per one neutron $/ \mathrm{cm}^{2}$.

Annealing within the $200 \ldots 500{ }^{\circ} \mathrm{C}$ interval leaves the EPR spectra of $3 \mathrm{C}-\mathrm{SiC}\langle n\rangle$ samples unaffected, qualitative and quantitative changes of them are evidenced after annealing at $600{ }^{\circ} \mathrm{C}$. From this point on, when the total concentration of defects becomes approximately one and a half times less mainly at the expense of the background line, the $T 1$ spectrum turns to the symmetric shape (Fig. 1). The following annealing step at $700{ }^{\circ} \mathrm{C}$ decreases the total concentration of defects more than an order of magnitude, the $T 1$ signal is dramatically diminished and completely disappears after the subsequent annealing at $800{ }^{\circ} \mathrm{C}$. The remainder of 
the $B G$ line includes at least two independent spectra, and it will no longer be discussed here. After annealing at $700{ }^{\circ} \mathrm{C}$, an axial spin $S=1 / 2$ defect with the $g$-tensor components $g_{\|}=2.0024, g_{\perp}=2.0039$ and principal axis along the $\langle 111\rangle$ crystal direction, labeled Ky6 and tentatively assigned to the negatively charged carbon vacancy-carbon antisite pair $\left(\mathrm{V}_{\mathrm{C}}-\mathrm{C}_{\mathrm{Si}}\right)^{-}$[20] is observed within the temperature range $77 \ldots 200 \mathrm{~K}$ as the dominant paramagnetic center (Fig. 2).

Contrary to the samples $3 \mathrm{C}-\mathrm{SiC}$ irradiated with electrons, appearance of the intense background line $B G$ in the 3C-SiC $\langle n\rangle$ samples should be related with the defect regions formed in the result of cascade displacements. Their first stage of annealing at $600{ }^{\circ} \mathrm{C}$ as well as considerable decrease in the intensity of the $B G$ spectrum correlate with annealing of partly amorphized regions in the $4 \mathrm{H}-\mathrm{SiC}$ sample implanted with aluminum ions [23] and theoretical calculations of defects thermal annealing mechanisms in heavy-ion irradiated 3C-SiC [24]. Further, the $B G$ line gradually decreases its intensity.

Among possible mechanisms of annealing inherent to silicon monovacancies, there theoretically considered were Frenkel pair recombination with silicon split interstitials and transformation of the silicon vacancy into a carbon vacancy-antisite complex $\mathrm{V}_{\mathrm{C}}-\mathrm{C}_{\mathrm{Si}}[16,25]$. However, any experimental confirmation was not reported up to date. In our case, the central part of the T1 spectrum is easily saturated even at room temperature, at the same time lines of the HF structure are saturated at one order higher powers. At $77 \mathrm{~K}$, the central part of the Tl spectrum is fully saturated, and only the lines of $\mathrm{HF}$ structure near the lines of hyperfine structure inherent to the $K y 6$ center can be registered (Fig. 2).

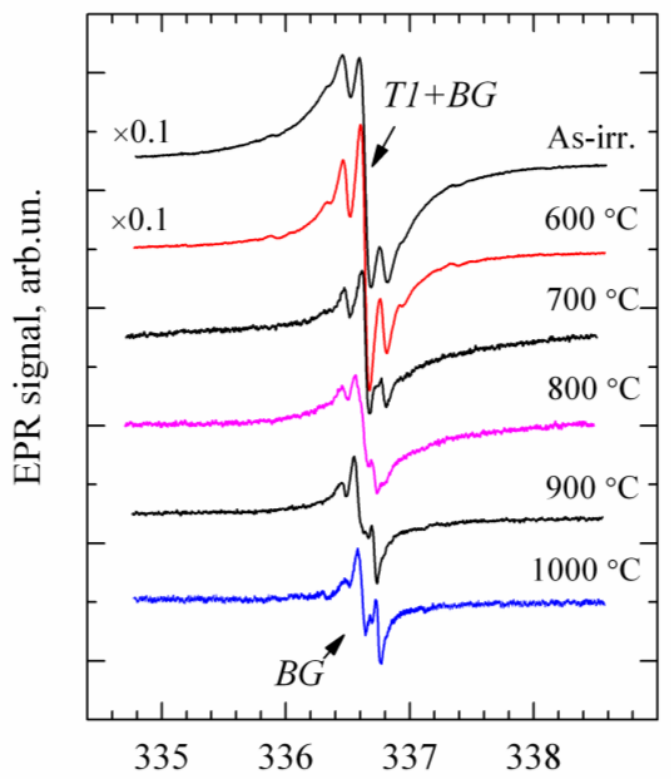

Fig. 1. EPR spectra of a $3 \mathrm{C}-\mathrm{SiC}\langle n\rangle$ sample before and after its step-by-step thermal annealing observed at $T=300 \mathrm{~K}$. The magnetic field direction $\mathbf{B}_{\mathbf{0}}$ is parallel to the [111] axis, microwave frequency $v=9.437 \mathrm{GHz}$.

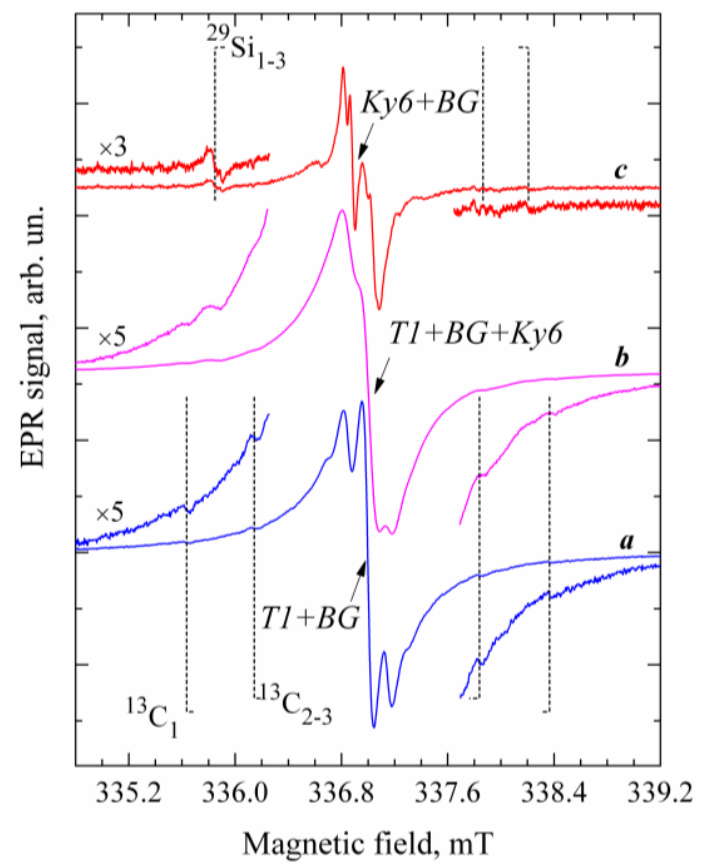

Fig. 2. EPR spectra of a $3 \mathrm{C}-\mathrm{SiC}\langle n\rangle$ sample before annealing $(a, b)$ measured at $T=320 \mathrm{~K}(a), T=77 \mathrm{~K}(b)$, and after annealing at $900{ }^{\circ} \mathrm{C}, T=77 \mathrm{~K}(c) ; \mathbf{B}_{0} \|[112], v=9.447 \mathrm{GHz}$. The dashed vertical lines represent positions of some $\mathrm{HF}$ structure lines of the $T 1$ and $K y 6$ centers.

Contrary to the EPR spectra obtained at room temperature after annealing of the samples at $700{ }^{\circ} \mathrm{C}$ (Fig. 1), the decrease in the intensity of lines $K y 6+B G$ at $77 \mathrm{~K}$ in the central part of the spectrum is insignificant, and the intensity of the $K y 6$ spectrum can be estimated with account of the intensity of lines in the HF structure. As seen from Fig. 3, the intensity of these lines grows doubly after annealing at $700{ }^{\circ} \mathrm{C}$, which can be indicative of partial transformation of the defects $T 1$ into the $K y 6$ defects. On the other hand, the possibility of the $\mathrm{V}_{\mathrm{Si}}{ }^{-}$transformation to a diamagnetic charge state of the $\mathrm{V}_{\mathrm{C}}-\mathrm{C}_{\mathrm{Si}}$ complex must not be ruled out. According to calculations within the $\mathrm{GW}$ approximations [25], the complex carries a $2^{+}$charge state in the major part of the band gap of $3 \mathrm{C}-\mathrm{SiC}$. Further, the Ky6 spectrum gradually decreases in its intensity but keeps observable even after annealing at $1100{ }^{\circ} \mathrm{C}$. The observed behavior of the $T 1$ centers during their annealing correlates with the results of theoretical calculations performed in the works $[16,17$, $25,26]$. In accord with hierarchy of annealing mechanisms, the high mobility of interstitial $\mathrm{C}$ and $\mathrm{Si}$ atoms, as compared to vacancies, provides their annealing at low temperatures. Transition of silicon vacancies from the metastable form to the stable configuration $\mathrm{V}_{\mathrm{C}}-\mathrm{C}_{\mathrm{Si}}$ requires to overcome a definite energy barrier, that is higher annealing temperature. 


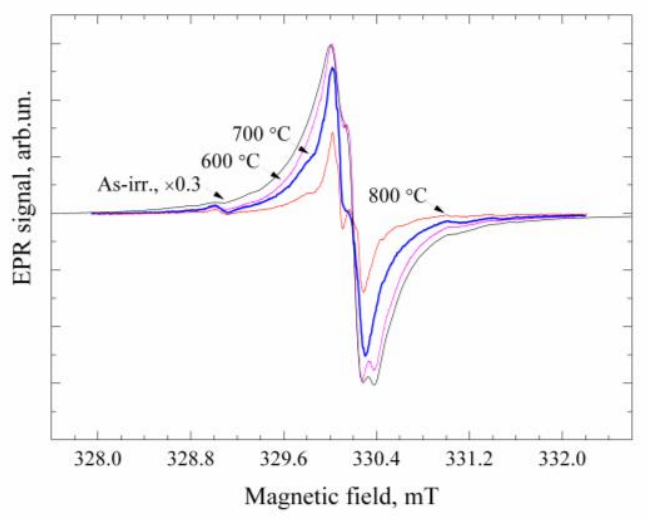

Fig. 3. EPR spectra of a $3 \mathrm{C}-\mathrm{SiC}\langle n\rangle$ sample before and after its step-by-step annealing up to $800{ }^{\circ} \mathrm{C} ; T=77 \mathrm{~K}, \mathbf{B}_{0} \|[112]$, $v=9.256 \mathrm{GHz}$.

\section{B. Neutral divacancy behavior with annealing}

In addition to the above-listed spectra, the components of the Ky5 center are observed with higher gain in the extended magnetic field range (Fig. 4). The initial concentration of this defect is found to be $3.0 \cdot 10^{17} \mathrm{~cm}^{-3}$. In a similar way to the $T 1$ center, changes in the $K y 5$ spectrum intensity start after annealing at $600{ }^{\circ} \mathrm{C}$. Then it becomes hardly visible after annealing at $700{ }^{\circ} \mathrm{C}$ and intensifies with full annealing of the silicon monovacancy at $800^{\circ} \mathrm{C}$. It should be noted that annealing of $\mathrm{V}_{\mathrm{Si}}$ is not accompanied by any additional growth in the intensity of the Ky5 divacancy spectrum. Simultaneously, a new spectrum labeled as $K y 5^{\prime}$ emerges with a higher value of the zero-field splitting than that for the Ky5 center, it becomes clearly determined after annealing at $900{ }^{\circ} \mathrm{C}$. With the following annealing step at $1000^{\circ} \mathrm{C}$, intensities of the $K y 5$ and $K y 5^{\prime}$ centers are strongly reduced and appearance of several additional lines with further enhanced zero-field splitting is observed.

A considerable decrease in the intensity of the Ky5 spectrum for the sample annealed at $700{ }^{\circ} \mathrm{C}$ and its further restoration after annealing at $800{ }^{\circ} \mathrm{C}$ can be related with changing the Fermi level. Indeed, as it will be shown below, after annealing at $700{ }^{\circ} \mathrm{C}$ additional lines from new paramagnetic defects appear in the EPR spectra. Besides, lowering the temperatures down to the helium ones, there can be detected the EPR lines belonging to the Ky7 centers that were attributed to negatively charged divacancies [19]. Appearance of new $K y 5^{\prime}$ centers can be related with mobility of divacancies at high annealing temperatures as well as their capture by impurities that occupy substitutional or interstitial positions in SiC lattice. It is significant to note that the intensity of the $K y 5^{\prime}$ spectrum grows at the expense of the $K y 5$ one after annealing at $900{ }^{\circ} \mathrm{C}$. The following "multiplication" of the $K y 5^{\prime}$ spectrum is probably related with capture of divacancies by other impurities. As it was shown using mass-spectrometry of secondary ions, beside the nitrogen impurity in irradiated $3 \mathrm{C}-\mathrm{SiC}\langle n\rangle$ crystals, there present are impurities of $\mathrm{Al}, \mathrm{P}$, In and some metals.

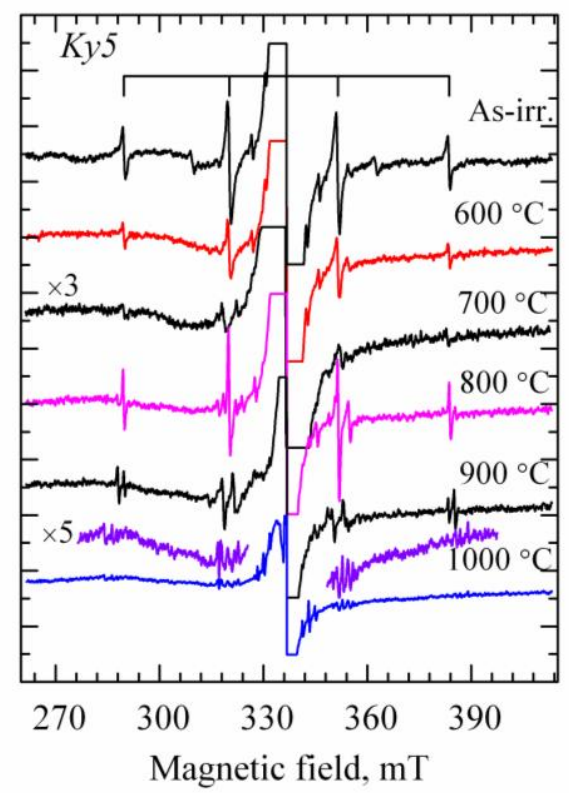

Fig. 4. EPR spectra of the $K y 5$ center in $3 \mathrm{C}-\mathrm{SiC}\langle n\rangle$ sample before and after its step-by-step annealing; $T=300 \mathrm{~K}$, $\mathbf{B}_{0} \|[111], v=9.437 \mathrm{GHz}$.

The $K y 5^{\prime}$ center, similar to the Ky5 one, possesses the $g$-value of 2.003 and can be described by the tensor of fine interaction with the direction of main axis along the $\langle 111\rangle$ crystal direction, however, with a little higher value of fine interaction constant $D$. Simultaneously with its appearance, one can observe lowering symmetry of the Ky5 center, when in addition to the axial component of crystalline field there appears the rhombic one. For the samples annealed at temperatures higher than $800{ }^{\circ} \mathrm{C}$, the spin Hamiltonian inherent to these centers looks as follows:

$\hat{H}=\hat{H}_{z}(\theta)+D\left(S_{z}^{2}-S(S+1)\right)+E\left(S_{x}^{2}-S_{y}^{2}\right)$.

Taking into account availability of magnetically non-equivalent positions in cubic crystal, this Hamiltonian is the matrix with the following diagonal elements:

$$
\begin{aligned}
& H_{11}=H(\theta)+0.5 D^{\prime}(\theta)-\varepsilon, \\
& H_{22}=-D^{\prime}(\theta)-\varepsilon, \\
& H_{33}=-H(\theta)+0.5 D^{\prime}(\theta)-\varepsilon .
\end{aligned}
$$

Where the Zeeman term should be written as

$$
H(\theta)=H\left(g_{\|} \cos ^{2}(\theta)+g_{\perp} \sin ^{2}(\theta)\right)
$$

and splitting in the zero field

$$
D^{\prime}(\theta)=\left\{\begin{array}{l}
\frac{1}{3}(D+E)\left(3 \cos ^{2}\left(\theta-54.7^{\circ}\right)-1\right), \\
\frac{1}{3}(D+E)\left(3 \cos ^{2}\left(\theta+54.7^{\circ}\right)-1\right), \\
\frac{1}{3}\left(D+\frac{1}{2} E\right) \sin ^{2}(\theta),
\end{array}\right.
$$


where $\varepsilon$ is the energy, and $\theta$ - the angle between direction of the magnetic field and the crystal axis $\langle 100\rangle$. The experimentally determined constants of the spin Hamiltonian for the center $K y 5$ are equal to $D=471 \cdot 10^{-4} \mathrm{~cm}^{-1}$ and $E=-11 \cdot 10^{-4} \mathrm{~cm}^{-1}$. The paramagnetic center $K y 5^{\prime}$ is described by slightly higher values of axial and rhombic constants $D=489 \cdot 10^{-4} \mathrm{~cm}^{-1}$ and $E=-19 \cdot 10^{-4} \mathrm{~cm}^{-1}$. Thus, the centers $K y 5$ and $K y 5^{\prime}$ possess the similar angle dependence of EPR spectra, and their radio-spectroscopic parameters differ only within $5 \%$.

We believe that this behaviour is an indication of local rearrangement in the neighborhood of divacancy and its intensity variations stem from a change of the Fermi-level position. Apparently, disappearance of the Ky5 EPR signal after annealing at 1000 and $1100{ }^{\circ} \mathrm{C}$ is an evidence of neutral divacancy recharging, since this defect has extremely high thermal stability in $\mathrm{SiC}$ [11].

\section{Carbon vacancy-related complex defects}

A considerable decrease in the intensity of the spectrum inherent to the primary defect $T 1$ after annealing at $700{ }^{\circ} \mathrm{C}$ is accompanied by appearance of new EPR spectra that belong to secondary defects. Available among them are lines of the known defect $T 6$ that was attributed to the spin-one vacancy-interstitial pair [12]. In $3 \mathrm{C}-\mathrm{SiC}\langle n\rangle$ samples, the $T 6$ spectrum has a much shorter spin-lattice relaxation time and can be readily picked out at higher level of the microwave power.

Contrary to irradiated with electrons epitaxial monocrystalline 3C-SiC films [12], the central lines in T6 spectra in the studied samples $3 \mathrm{C}-\mathrm{SiC}\langle n\rangle$ are observed along with several pairs of satellite lines (Fig. 5). Like to spectra of negatively charged carbon vacancies $\mathrm{V}_{\mathrm{C}}^{-}$with the spin $S=1 / 2$ in the polytype $4 \mathrm{H}-\mathrm{SiC}$ [13], the ratio of intensities of satellite lines for $\mathrm{Si}_{1}$ and $\mathrm{Si}_{2-4}$ to that of the central line reaches the values 5 and $13 \%$, respectively. The pairs of lines with the splitting similar to those of $\mathrm{Si}_{2-4}$ lines are also observed both on low-field and high-field wings of the central part in the spectrum belonging mainly to the $K y 6$ defect. Our analysis of the EPR spectra showed that these pairs cannot be related with the spectrum of the Ky6 centers. Besides, changes in the intensity of these lines during annealing of the samples correlate with changes in the intensity of EPR lines related to the T6 spectrum with account of their possible association.

Making assumption that these lines are related to the lines of HF structure concerned with the central transition $1 / 2 \leftrightarrow-1 / 2$ of the defect with the spin $S=3 / 2$, we performed description of the line positions for experimental EPR spectra. As it was ascertained, the latter are considerably better described by Hamiltonian (1) with the spin $S=3 / 2$ than with the spin $S=1$ (Fig. 4) as well as parameters $g_{\|}=2.0021, g_{\perp}=2.0044$ and the principal value of the fine interaction tensor $D=6.53 \mathrm{mT}$ $(183 \mathrm{MHz})$ oriented along the $\langle 111\rangle$ direction. The values of $\mathrm{HF}$ splitting constants are equal for one silicon atom $\mathrm{Si}_{1}$ $A_{\|}=1.08 \mathrm{mT}(30.1 \mathrm{MHz}), A_{\perp}=0.85 \mathrm{mT}(23.8 \mathrm{MHz})$ and for three equivalent silicon atoms $\mathrm{Si}_{2-4} A_{\|}=5.88 \mathrm{mT}$ $(164.8 \mathrm{MHz}), A_{\perp}=4.25 \mathrm{mT}(119.1 \mathrm{MHz})$. In this case, the principal axes of the HF interaction tensors are also oriented along the $\langle 111\rangle$ direction. Since the isolated carbon vacancy has the spin $S=1 / 2$ and as a negative- $U$ center is detectable only under optical excitation [13], we should make the assumption that the T6 defect is complex and consists of the pair $\mathrm{V}_{\mathrm{C}}-\mathrm{X}$ with the spin $S=3 / 2$, where the letter $\mathrm{X}$ designates still unknown atom in the vicinity of $\mathrm{V}_{\mathrm{C}}^{-}$. As it was predicted earlier [12], it may be the atom $\mathrm{C}$ or $\mathrm{Si}$ in the nearest tetrahedral interstitial position. These defects possess high thermal stability. The intensity of the T6 center EPR lines grows after annealing at temperatures from $T_{\text {ann }}=700{ }^{\circ} \mathrm{C}$ up to $900{ }^{\circ} \mathrm{C}$ and remains practically unchanged after following annealing at the temperature $T_{\mathrm{ann}}=1100{ }^{\circ} \mathrm{C}$. It means that transition of the $\mathrm{X}$ atom into the vacancy position is separated with a highenergy barrier. In general, ascertaining the nature of $\mathrm{X}$ atoms requires additional investigations by using ENDOR and theoretical calculations.

\section{Carbon $\langle 100\rangle$ split interstitials}

Simultaneously with appearance of spectra corresponding to the $T 6$ defect, the intensity of EPR lines related with new anisotropic centers is considerably increased (Fig. 6). The observed lines can be represented as superposition of two spectra for $K y 8$ and $K y 8^{\prime}$ centers that possess the electron spin $S=1$ and are characterized by insignificant differences in the value of the fine structure constant $D$. The direction of the principal axis for the tensor of fine interaction coincides with the $\langle 100\rangle$ direction in crystal lattice.

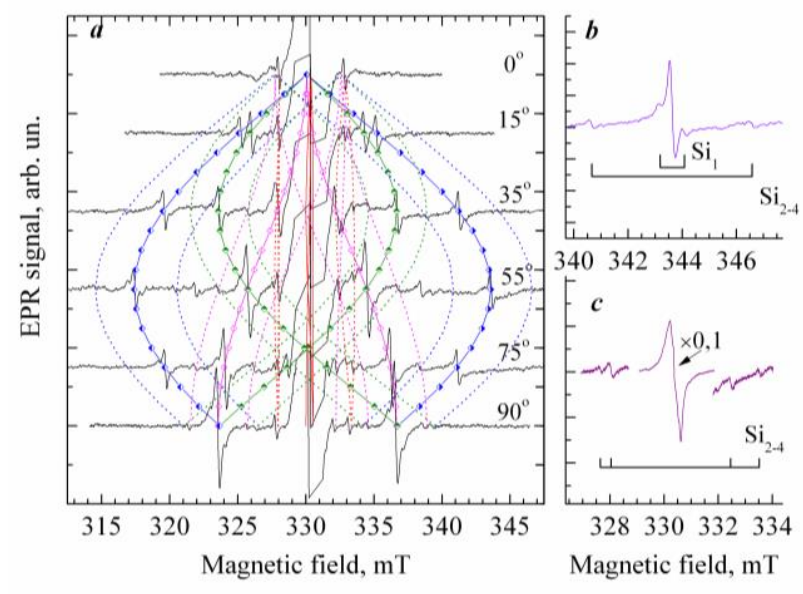

Fig. 5. a) EPR spectra of the $T 6$ center measured at $T=77 \mathrm{~K}$ and $v=9.264 \mathrm{GHz}$ for a rotation of the magnetic field in the (1 $1 \overline{1} 0)$ plane, $\theta=0^{\circ}$ corresponds to the [001] crystal direction. The symbols present experimental line positions, solid and dashed lines are calculated according to the parameters listed in the text. b) Hyperfine lines of the high-field component of the $T 6$ spectrum, $\theta=55^{\circ}$. c) Hyperfine lines attributed to the $(1 / 2 \leftrightarrow-1 / 2)$ transition of the spin $S=3 / 2 T 6$ center, $\theta=55^{\circ}$. The latter is hidden under more intense $K y 6$ spectrum which is disturbed by high level of microwave power and modulation amplitude. 


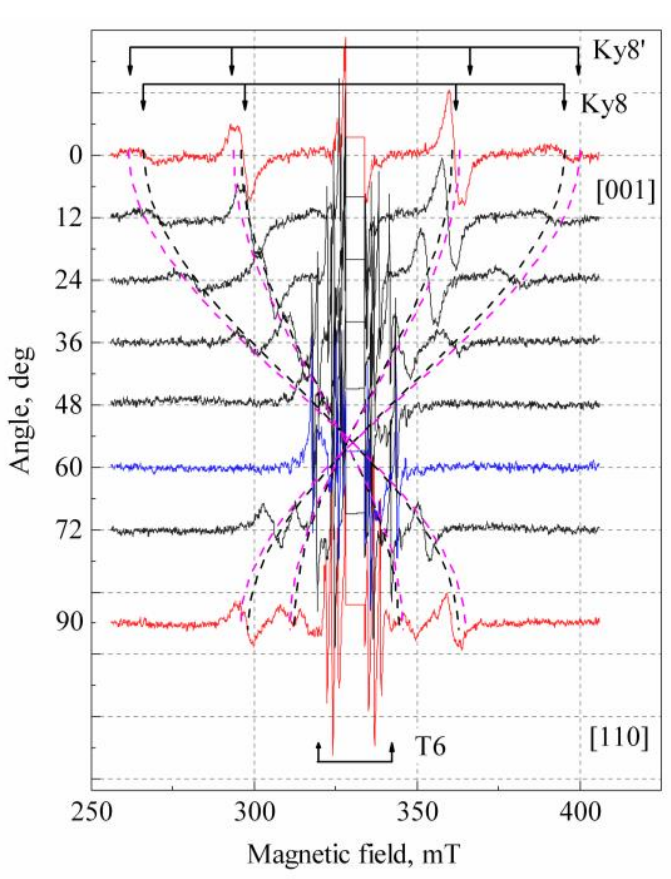

Fig. 6. Angular dependence of the $K y 8$ and $K y 8^{\prime}$ spectra for a $3 \mathrm{C}-\mathrm{SiC}\langle n\rangle$ sample annealed at $900^{\circ} \mathrm{C}, \quad T=77 \mathrm{~K}$, $v=9.264 \mathrm{GHz}$. The dashed lines are calculated according to the parameters presented in the text.

The resonance magnetic fields were found using the following expression:

$h v=g \beta H+f\left(D, \frac{D^{2}}{g \beta H}\right)$,

where the first term is related with Zeeman splitting, while the second one describes fine splitting with account of the second order correction. Being based on calculations $[27,28]$ and using the spin value $S=1$, the expression for calculation of the angular dependence for EPR lines can be simplified, and resonance positions of the lines can be described by the formula:

$H_{r}=H_{0}+D\left(3 \cos ^{2} \theta-1\right)-\frac{D^{2}}{8 H_{0}}\left(\sin ^{2} 2 \theta+\sin ^{4} \theta\right)$,

where $H_{r}$ is the resonance position of EPR line, $H_{0}-$ magnetic field value that corresponds to the Zeeman term, and the second and third terms describe fine splitting and the second order correction.

Fig. 6 shows the experimentally obtained at $77 \mathrm{~K}$ angular dependence for EPR lines of the sample annealed at the temperature $900{ }^{\circ} \mathrm{C}$. The paramagnetic centers $K y 8 / K y 8^{\prime}$ possess the same symmetry, $g$-factor and direction of the $D$-tensor axis and differ only by the values of their fine-splitting constants: $g=$ $2.000 \pm 0.010, D \approx 589 \cdot 10^{-4} \mathrm{~cm}^{-1}$ for $K y 8$ and $D \approx$ $646 \cdot 10^{-4} \mathrm{~cm}^{-1}$ for $K y 8^{\prime}$ at $T=77 \mathrm{~K}$. These values are close by their order to that calculated from the first principles $D$-constant for the $\langle 100\rangle$ carbon split interstitial $(\mathrm{CC})_{\mathrm{C}}$ (i.e., two carbon atoms that share one $\mathrm{C}$-site) in $3 \mathrm{C}-\mathrm{SiC}$ in the neutral charge state
$|D|=670 \cdot 10^{-4} \mathrm{~cm}^{-1}[29]$. Thus, like to the case of divacancies $K y 5 / K y 5^{\prime}$, the spectra of $K y 8 / K y 8^{\prime}$ centers belong to two similar defects.

When analyzing diffusion of nitrogen atoms, beside the $(\mathrm{CC})_{\mathrm{C}}$ defect, the authors of [30] considered the pair of atoms $(\mathrm{NC})_{\mathrm{C}}$ that also forms the interstitial defect split in the direction $\langle 100\rangle$. Since the $D$-constant value is determined both by dipole-dipole and spin-orbital interactions [28], and the latter is higher for nitrogen atoms, then the $K y 8$ spectrum is tentatively related by us with the pair $(\mathrm{CC})_{\mathrm{C}}$, while the $K y 8^{\prime}$ spectrum - with the split interstitial $(\mathrm{NC})_{\mathrm{C}}$. It should be noted that the EPR lines of $K y 8 / K y 8^{\prime}$ centers have low intensity also in unannealed samples, which means that they are primary defects in $3 \mathrm{C}-\mathrm{SiC}\langle\mathrm{n}\rangle$, they considerably grow after annealing at $T_{\mathrm{ann}}=900{ }^{\circ} \mathrm{C}$ and are doubly decreased after annealing at $T_{\mathrm{ann}}=1100^{\circ} \mathrm{C}$.

\section{Conclusions}

Thus, thermal annealing of $3 \mathrm{C}-\mathrm{SiC}\langle n\rangle$ samples enabled to find several temperatures that provide annealing and transformation of primary defects, i.e., $\mathrm{V}_{\mathrm{Si}}{ }^{-}$, as well as pairs $\left(\mathrm{V}_{\mathrm{C}}-\mathrm{C}_{\mathrm{Si}}\right)^{-},\left(\mathrm{V}_{\mathrm{Si}}-\mathrm{V}_{\mathrm{C}}\right)^{0}$ and $(\mathrm{CC})_{\mathrm{C}}{ }^{0}$. At $T_{\text {ann }}=600{ }^{\circ} \mathrm{C}$, there takes place annealing of defects of the vacancy type and broken bonds in the regions related with cascade displacements and mainly conditioned by a high mobility of interstitial atoms. Annealing of negatively charged silicon monovacancies $V_{\mathrm{Si}}{ }^{-}$takes place within the temperature interval $T_{\mathrm{ann}}=700 \ldots 800^{\circ} \mathrm{C}$, the partial transformation $V_{\mathrm{Si}}{ }^{-} \rightarrow\left(V_{\mathrm{C}}-C_{\mathrm{Si}}\right)^{-}$has been registered in this interval. Appearance of the $T 6$ centers after annealing within this interval is indicative of formation of pairs with intrinsic atoms or impurities being one of the possible ways for $V_{\mathrm{C}}$ transformation in the $3 \mathrm{C}-\mathrm{SiC}\langle n\rangle$ samples.

As concerning divacancies, their detection essentially depends on position of the Fermi level in annealed samples, and lowering symmetry after annealing at $T_{\text {ann }}=800^{\circ} \mathrm{C}$ can be related with capture of these defects by impurity atoms. It should be also noted that for all the above defects, starting from $T_{\text {ann }}=$ $600{ }^{\circ} \mathrm{C}$, one can observe gradual narrowing of the EPR lines, which is indicative of progressive ordering the structure of irradiated samples.

\section{Acknowledgment}

The authors are grateful to the Center of Shared Research Equipment of Institute of Magnetism of the National Academy Science of Ukraine for placing at their disposal the EPR spectrometer for some temperature measurements.

\section{References}

1. Silicon Carbide: Recent Major Advances, ed. by W.J. Choyke, H. Matsunami, G. Pensl. Springer Berlin, Heidelberg, 2004. 
2. Silicon Carbide, V. 2: Power Devices and Sensors, ed. by P. Friedrichs, T. Kimoto, L. Ley, G. Pensl. Wiley-VCH Verlag GmbH, Weinheim 2011.

3. J.M. Spaeth, J.R. Niklas, R.H. Bertram, Structural Analysis of Point Defects in Solids. Springer Berlin, 1992.

4. V.Ya. Bratus', T.T. Petrenko, S.M. Okulov, T.L. Petrenko, Positively charged carbon vacancy in three inequivalent lattice sites of $6 \mathrm{H}-\mathrm{SiC}$ : combined EPR and density functional theory study // Phys. Rev. B, 71(12), 125202 (2005).

5. W.F. Koehl, B.B. Buckley, F.J. Heremans, G. Calusine, D. Awschalom, Room temperature coherent control of defect spin qubit in silicon carbide // Nature, 479, p. 84-87 (2011).

6. L.A. de S. Balona, J.H.N. Loubser, ESR in irradiated silicon carbide // J. Phys. C: Solid State Phys. 3, p. 2344-2351 (1970).

7. N.M. Pavlov, M.I. Iglitsyn, M.G. Kasaganova, V.N. Solomatin, Centers having spin 1 in silicon carbide irradiated with neutrons and $\alpha$-particles // Sov. Phys. Semiconductors, 9, p. 845-849 (1975).

8. T. Wimbauer, B.K. Meyer, A. Hofstätter, A. Scharmann, H. Overhof, Negatively charged Si vacancy in $4 \mathrm{H} \mathrm{SiC}$ : A comparison between theory and experiment // Phys. Rev. B, 56, p. 7384-7388 (1997).

9. H.J. von Bardeleben, J.L. Cantin, I. Vickridge, G. Battistig, Proton-implantation-induced defects in $n$-type $6 \mathrm{H}-$ and $4 \mathrm{H}-\mathrm{SiC}$ : An electron paramagnetic resonance study // Phys. Rev. B, 62, p. 1012610134 (2000).

10. H.J. von Bardeleben, J.L. Cantin, L. Henry, M.F. Barthe, Vacancy defects in $p$-type $6 \mathrm{H}-\mathrm{SiC}$ created by low-energy electron irradiation // Phys. Rev. B, 62, p. 10841-10846 (2000).

11. J. Isoya, T. Umeda, N. Mizuochi, N.T. Son, E. Janzen, T. Ohshima, EPR identification of intrinsic defects in SiC // physica status solidi (b), 245, p. 1298-1314 (2008).

12. H. Itoh, A. Kawasuso, T. Ohshima et al., Intrinsic defects in cubic silicon carbide // physica status solidi (a), 162, p. 173-198 (1997).

13. X.T. Trinh, K. Szasz, T. Hornos, K. Kawahara, J. Suda, T. Kimoto, A. Gali, E. Janzen, and N.T. Son, Negative-U carbon vacancy in $4 \mathrm{H}-\mathrm{SiC}$ : Assessment of charge correction schemes and identification of the negative carbon vacancy at the quasicubic site // Phys. Rev. B, 88, 235209 (2013).

14. V.S. Vainer, V.A. Il'in, EPR of exchanged-coupled vacancy pairs in hexagonal silicon carbide // Sov. Phys. Solid State, 23, p. 2125-2131 (1981).

15. N.T. Son, E. Janzen, J. Isoya et al., Identification of a Frenkel-pair defect in electron-irradiated 3C SiC // Phys. Rev. B, 80, 125201 (2009).

16. M. Bockstedte, A. Mattausch, O. Pankratov, $A b$ initio study of the migration of intrinsic defects in 3C-SiC // Phys. Rev. B, 68, 205201 (2003).
17. M.J. Zheng, N. Swaminathan, D. Morgan, I. Szlufarska, Energy barriers for point-defect reactions in 3C-SiC // Phys. Rev. B, 88, 054105 (2013).

18. I.V. Ilyin, M.V. Muzafarova, E.N. Mokhov, P.G. Baranov, Electron paramagnetic resonance studies of multi-defect clusters in neutron irradiated silicon carbide // Semicond. Sci. Technol. 22, p. 270-278 (2007).

19. V.Ya. Bratus', R.S. Melnik, S.M. Okulov, V.N. Rodionov, B.D. Shanina, M.I. Smoliy, A new spin one defect in cubic SiC // Physica B, 404, p. 4739-4741 (2009).

20. V. Bratus', R. Melnik, S. Okulov, B. Shanina, V. Golub, I. Makeeva, An EPR study of defects in neutron-irradiated cubic SiC crystals // Mater. Sci. Forum, 740-742, p. 361-365 (2013).

21. S.N. Gorin, L.M. Ivanova, Cubic silicon carbide (3C-SiC): structure and properties of single crystals grown by thermal decomposition of methyl trichlorosilane in hydrogen // physica status solidi (b), 202, p. 221-245 (1997).

22. F. Maekawa, K. Ochiai, K. Shibata, Y. Kasugai, M. Wada, Y. Morimoto, H. Takeuchi, Benchmark experiment on silicon carbide with $\mathrm{D}-\mathrm{T}$ neutrons and validation of nuclear data libraries // Fusion Eng. and Design, 58-59, p. 595-600 (2001).

23. Y. Zhang, W.J. Weber, W. Jiang, A. Hallén, G. Possnert, Damage evolution and recovery on both $\mathrm{Si}$ and $\mathrm{C}$ sublattices in $\mathrm{Al}$-implanted $4 \mathrm{H}-\mathrm{SiC}$ studied by Rutherford backscattering spectroscopy and nuclear reaction analysis // J. Appl. Phys. 91, p. 6388 (2002).

24. D. Guo, I. Martin-Bragado, C. He, H. Zang, P. Zhang, Modeling of long-term defect evolution in heavy-ion irradiated 3C-SiC: Mechanism for thermal annealing and influences of spatial correlation // J. Appl. Phys. 116, 204901 (2014).

25. F. Bruneval, G. Roma, Energetics and metastability of the silicon vacancy in cubic SiC // Phys. Rev. B, 83, 144116 (2011).

26. M. Bockstedte, A. Mattausch, O. Pankratov, $A b$ initio study of the annealing of vacancies and interstitials in cubic SiC: Vacancy-interstitial recombination and aggregation of carbon interstitials // Phys. Rev. B, 69, 235202 (2004).

27. B. Bleaney, D.J.E. Ingram, The paramagnetic resonance spectra of two salts of manganese // Proc. Roy. Soc. Lond. A, 205, p. 336-356 (1951).

28. A. Abragam, B. Bleaney, Electron Paramagnetic Resonance of Transition Ions. V. 1, M., Mir, 1972.

29. T.T. Petrenko, T.L. Petrenko, V.Ya. Bratus', The carbon $\langle 100\rangle$ split interstitial in $\mathrm{SiC} / /$ J. Phys.: Condens. Matter. 14, p. 12433-12440 (2002).

30. U. Gerstmann, E. Rauls, Th. Frauenheim, and H. Overhof, Formation and annealing of nitrogenrelated complexes in SiC // Phys. Rev. B, 67, p. 205202 (2003). 